\title{
Estado, apropiación social de las tecnologías de la información y comunicación y pobreza
}

\section{State, social appropriation of information and communication technologies and poverty}

\author{
doi: http://dx.doi.org/10.32870/ \\ espiral.v25i73.7010
}

\section{Resumen}

Este artículo presenta una aproximación teórica sobre el papel del Estado mexicano en la apropiación social de las tecnologías de la información y comunicación (TIC), como parte de una investigación más amplia que analiza dicho fenómeno empíricamente. El marco de análisis presentado sugiere que cuando la tecnología es adoptada a gran escala por la sociedad, se convierte en parte del marco institucional y organizativo del Estado, por lo que el propio Estado puede impedir o reproducir problemas sociales como la pobreza a través de ella. En este sentido, se concluye que se requieren análisis institucionales de los Estados que permitan coadyuvar en la construcción de capacidades para promover la apropiación social de las TIC y disminuir problemáticas sociales como la pobreza.

Palabras clave: Estado, apropiación social, TIC, México, pobreza.

\author{
Christian I. Becerril-Velasco
}

\begin{abstract}
This article aims to present a theoretical approach to the role of the Mexican state in the social appropriation of information and communication technologies (ICTs) and the reduction of poverty, as part of a broader research whose objective is to empirically analyze that role. The framework of analysis presented suggests that when technology is appropriated on a large scale by society, it becomes part of the institutional and organizational framework of the state, and because of that the state itself can prevent or reproduce social problems such as poverty through it. In this line of thinking, we conclude that institutional analyzes of the states are required to be able to contribute to the construction of capacities to promote the social appropriation of ICTS and reduce social problems such as poverty.
\end{abstract}

Keywords: State, social appropriation, ICTs, Mexico, poverty.

\footnotetext{
-Investigador del Centro de Investigación e Innovación en Tecnologías de la Información y Comunicación (INFOTEC), México. ORCID: http://orcid.org/0000-0003-I423-4I50

christian.becerril@infotec.mx

Fecha de recepción: 17 de octubre de 2017. Fecha de aceptación: 09 de julio de 2018.
} 


\section{Introducción}

El fuerte vínculo que existe entre el desarrollo de las tecnologías de la información y comunicación (TIC), el crecimiento económico y, en consecuencia, la reducción de la pobreza ha sido ya ampliamente discutido (Rosenberg y Trajtenberg, 2004; Ristuccia y Solomou, 2010; Galperin y Fernanda Viecens, 2017; Nord, Riggio, y Paliszkiewicz, 2017). De igual forma, en los últimos años se ha demostrado que en los países más desarrollados la simbiosis entre la formación de capacidades institucionales internas y la apertura económica ha sido clave en el éxito de la apropiación social de las TIC para reducir la pobreza (Gillwald, 2010; Lopez-Aymes, 2010). Sin embargo, no se han explicado del todo las razones del impedimento de los países en vías de desarrollo para alcanzar los logros que se han presentado en otras partes del mundo en cuanto a la apropiación social de las TIC para reducir la pobreza.

Como parte de una investigación más amplia cuyo objetivo es analizar empíricamente el papel del Estado mexicano en la apropiación social de las TIC y la reducción de la pobreza, el presente artículo tiene la finalidad de presentar una aproximación teórica sobre el tema. Este documento se divide en cinco secciones: en la primera, se explica la relación entre el conjunto institucional y organizacional del Estado y las TIC; en la segunda, se reflexiona sobre la importancia de la intervención del Estado para la adopción de las TIC; en la tercera sección, se explora el concepto de la apropiación de las TIC como una fase ulterior a la aplicación de las mismas; en la cuarta sección, se discute el papel del Estado en la reducción de la pobreza a través de las TIC; y finalmente, en la quinta sección, se presentan las principales conclusiones. 


\section{Estado, instituciones y tecnologías de la información y comunicación}

La importancia del estudio de las TIC radica en el hecho de que se tratan del tipo de tecnologías que aparecen cada cuarenta o cincuenta años, impactan todos y cada uno de los sectores de la economía y transforman la forma en que las organizaciones (públicas y privadas) y las personas persiguen sus metas y objetivos. ${ }^{1}$

Llamadas por algunos tecnologías de propósito general (GPT, por sus siglas en inglés) (Jovanovic y Rousseau, 2005), paradigmas tecnoeconómicos (Freeman y Perez, 1988) o transformaciones tecnológicas (Standing, 2010), se propagan a lo largo de la economía y desempeñan el papel de tecnologías habilitadoras, pues abren oportunidades de innovación y desarrollo (Bresnahan y Trajtenberg, 1995; Bresnahan, 2002). ${ }^{2}$ Tal ha sido el caso de las máquinas de vapor, la electricidad, la tecnología desarrollada para explotar el petróleo y las TIC. ${ }^{3}$

I.De acuerdo con Perez (1983), el sistema capitalista se divide en etapas delineadas por tecnologías específicas alrededor de las cuales ocurre el crecimiento de la productividad dentro y entre las empresas, los giros económicos y los países.

2. Los representantes de las GPT señalan que estas deben cumplir con al menos tres características: I) difusión generalizada, pues deben impactar los diferentes sectores de la economía; 2) magnitud, pues deben mejorar con el tiempo y reducir los costos para los usuarios; y 3) facilitar la invención, pues deben impulsar el desarrollo de tecnologías complementarias (Rosenberg y Trajtenberg, 2004; Ristuccia y Solomou, 2010; Bresnahan, 2010).

3. Freeman y Perez (1988) señalan que han existido cinco paradigmas tecnoeconómicos: la mecanización de la industria con base en el acero, las máquinas de vapor, la electricidad, la producción en masa con derivados del petróleo y las TIC. Por otra parte, los estudiosos de las GPT señalan solamente los cuatro últimos (Jovanovic y Rousseau, 2005; Bresnahan y Trajtenberg, 1995).Algunos otros señalan que la tecnología desarrollada para la agricultura ocasionó la primera revolución económica (North, 1994). Independientemente de las variaciones entre las diferentes clasificaciones, todas señalan a las TIC como el más reciente cambio tecnológico que ha impactado todos y cada uno de los sectores de la economía y transformado las rutinas de la sociedad en general. 
Durante la segunda mitad del siglo XIX, la industrialización y los niveles de desarrollo económico que lograron Gran Bretaña, Alemania y la mayoría de los países europeos fueron atribuidos principalmente a la apropiación de tecnología de punta, en ese entonces las máquinas de vapor. ${ }^{4}$ En este sentido, la formula parecía sencilla: los países en vías de desarrollo debían adoptar las tecnologías utilizadas por los países desarrollados e industrializarse para lograr desarrollo económico y social (Gerschenkron, 1962; Neüman, 2008). En consecuencia, este esquema fue reproducido, sin apartarse de lo previsto, por la gran mayoría de los países en vías de desarrollo durante la mayor parte del siglo XX.

Los países latinoamericanos no fueron ajenos a ello. Sin embargo, no se tomó en cuenta que la tecnología es difícilmente adaptada de manera inmediata, y que sus efectos sobre la economía están condicionados a diversos factores, como las circunstancias macroeconómicas y los entornos institucionales y culturales (David, 1989; Galperin, 2010). De hecho, en los últimos años se ha demostrado que los países con instituciones fuertes experimentan mayores tasas de crecimiento económico y de adopción de tecnología y, por lo tanto, son aquellos que cierran más rápidamente las diferentes brechas de atraso en relación con los países más desarrollados (Manca, 2010; Lopez-Aymes, 2010; Evans y Sewell, 2013).

Corea del Sur, por ejemplo, es uno de los países con mayor crecimiento económico, disminución de la pobreza y adopción de tecnología en las últimas décadas debido, entre otras cosas, al fortalecimiento de su aparato institucional y organizativo. La intervención del Estado surcoreano en

\footnotetext{
4. Aunque las máquinas de vapor generaron la primera Revolución Industrial, a mediados del siglo XIX, su aparición data de inicios del siglo XVIII. Por su parte, la electricidad fue implementada alrededor de 1895, la tecnología desarrollada para explotar el petróleo a finales de los años veinte y las TIC a principios de 1970 con la invención del primer microprocesador (Simon, 1987; Rousseau, 2008).
}

\section{0}


la expansión de su infraestructura de telecomunicaciones entre los años ochentas y noventas -en contra de la ideología neoliberal, que pugna por la privatización de este tipo de servicios- le ha permitido tener una cobertura de casi $100 \%$ en el acceso a TIC como el internet y la telefonía fija y móvil (Unión Internacional de Telecomunicaciones, 2017).

Más que eso, a lo largo de casi cuatro décadas, el Estado surcoreano ha invertido fuertemente en investigación y desarrollo, educación y capacitación tecnológica universal, y creado institutos y organizaciones públicas especializadas, con lo que ha incrementado las oportunidades de su población para afrontar los retos que la más reciente revolución tecnológica ha presentado: entre otros, el combate a la pobreza (Lopez-Aymes, 2010; Boncheva, Licona Michel, Loaiza Becerra, et al., 2016). ${ }^{5}$ La idea principal detrás del ajuste de su marco institucional para promover la adopción y apropiación de las TIC fue adaptarse rápidamente a la llamada sociedad del conocimiento y la información, en la cual las TIC son vitales.

En este orden de ideas, el funcionamiento de una economía, entonces, es resultado de sus instituciones y la tecnología desarrollada, por lo que existe una compleja interdependencia entre el cambio institucional y el tecnológico a medida que la economía evoluciona. El marco institucional y la tecnología desarrollada delimitan los costos de transacción, es decir, los costos de realizar intercambios económicos; a su vez, el marco institucional y los costos de transacción determinan el grado de éxito de cualquier tecnología (North, 1994). Esta interdependencia se complejiza aún más si tomamos en cuenta que, como lo pone Saviotti (2005), las instituciones necesarias para promover el desarrollo de una

5. Lopez-Aymes (2010) ofrece una explicación completa de cómo Corea del Sur fortaleció su marco institucional para promover la adopción y apropiación de las TIC. 
determinada tecnología pueden constituir un obstáculo para su posterior cambio o reemplazo por tecnologías más nuevas.

Perez (2013) arguye que las nuevas dinámicas introducidas por la tecnología reemplazante producen una perturbación cada vez mayor en la evolución de los mercados, lo que hace que se transformen gradualmente el tejido social y los mecanismos de generación tecnológica. ${ }^{6}$ La introducción de nuevas tecnologías, entonces, tiene efectos tan fuertes en la economía que su difusión debe ser acompañada de ajustes estructurales que incluyan cambios institucionales y sociales a nivel nacional e internacional para lograr un emparejamiento entre la nueva tecnología y el régimen económico social presente (Freeman y Perez, 1988). ${ }^{7}$ Se crea así un proceso de destrucción creativa tanto a nivel económico, pues se cambia la manera de hacer negocios, como institucional, ya que se debe modificar la estructura organizacional del Estado. ${ }^{8}$

Este reacomodo del marco institucional del Estado permea y delinea poco a poco las prácticas culturales de la población, pues le permite apropiarse de la tecnología y hacer uso de ella en su vida diaria (Perez, 1983). En este sentido, la adopción de tecnologías de la magnitud de las

\footnotetext{
6.A manera de ejemplo, David (1989) señala que las últimas y primeras décadas del siglo XX comparten el haber visto el cambio de una tecnología a otra con una crisis económica aparejada; sin embargo, los años subsecuentes fueron de crecimiento acelerado en los países más desarrollados.

7. North (1994) explica que las innovaciones institucionales que disminuyeron los costos de transacción en la etapa previa a la revolución de las máquinas de vapor fueron aquellas que incrementaron la movilidad de capital, disminuyeron los costos de información y permitieron compartir los riesgos y mejorar el cumplimiento de la ley. Por otra parte, los derechos de propiedad bien especificados y aplicados, la descentralización de las decisiones políticas y económicas y una competencia efectiva fueron parte de la estructura institucional que las economías modernas utilizaron para sostener la productividad de la primera Revolución Industrial (máquinas de vapor).

8. Según Perez (1983), las empresas que pueden escapar de esta destrucción creativa o crisis son las que están vinculadas a la producción o utilización de la nueva tecnología, y es hacia estas áreas a donde tiende a ir la nueva inversión.
} 
TIC o la electricidad puede ser considerada como la de un conjunto de rutinas o prácticas tecnológicas compartidas por organizaciones (públicas y privadas) y por personas, las cuales establecen patrones de comportamiento humano que, si se adoptan a gran escala, se convierten en instituciones (Saviotti, 2005). No obstante, es complicado saber qué tipo de instituciones se requieren o hasta qué punto pueden cambiar las rutinas o prácticas de las personas $u$ organizaciones a través del uso y apropiación de las TIC.

Saviotti (2005) señala que la creación de las instituciones que se requieren para apuntalar el desarrollo de la tecnología tiene que ser estudiada a lo largo del avance de la tecnología misma. Esto se debe principalmente al hecho de que cuando la tecnología remodela la sociedad no es resultado de una sola invención, sino de innovaciones tecnológicas complementarias que van apareciendo tanto en los países creadores de dicha tecnología como en los países importadores (Perez, 1983; Simon, 1987). Así, las instituciones se crearán normalmente en las primeras etapas de la difusión de la tecnología, es decir, cuando esta recién aparece, y su grado de difusión será determinado por la presencia (o ausencia) de tales instituciones.

Según Hall y Khan (2003), la difusión de la tecnología, más que su invención o innovación, determina el ritmo de crecimiento económico y la tasa de productividad, pues mientras que la tecnología no es ampliamente adoptada contribuye poco al bienestar de las personas. ${ }^{9}$ A nivel internacional, la apertura económica es quizás la fórmula que facilita en mayor medida la transferencia tecnológica y estimula la innovación (Fagerberg, Srholec, y Verspagen, 2010;

9. De acuerdo a Hall y Khan (2003), la difusión es el resultado acumulado o agregado de una serie de cálculos individuales que sopesan los beneficios de adoptar una tecnología contra los costos de cambio, normalmente en un ambiente caracterizado por incertidumbre e información limitada sobre los beneficios y costos de la tecnología. 
Qiu y Cantwell, 2016). Sin embargo, se requieren también cambios institucionales a nivel nacional o local, ya que, como se mencionó anteriormente, los Estados con instituciones fuertes cierran más rápidamente las diferentes brechas de atraso en relación con los países más desarrollados.

Hasta aquí, se ha dicho que cuando la tecnología se adopta a gran escala se convierte en parte del marco institucional y organizacional del Estado y que, en consecuencia, un conjunto de instituciones y organizaciones fuertes puede contribuir a combatir problemas sociales como la pobreza a través de las TIC, como ocurrió en el caso de Corea del Sur.

En el siguiente apartado, se aborda brevemente la forma en que la introducción de nuevas tecnologías puede incrementar las desigualdades económicas en una sociedad, por lo que es primordial que el Estado intervenga en la formación de un marco institucional y organizacional que permita su adopción por parte de los organismos públicos y privados y las personas para evitar y paliar problemas sociales como la pobreza.

\section{La importancia de la intervención del Estado en la adopción de las tecnologías de la información y comunicación}

Durante la Revolución Industrial, en el siglo XIX, resultada de la invención de las máquinas de vapor en Inglaterra, se presentaron cambios estructurales tanto a nivel de dicha nación como internacional incitados por los países en desarrollo que buscaban aprovechar al máximo las oportunidades de crecimiento que la tecnología ofrecía. El problema fue, como arguyó Polanyi (2001), que el Gobierno inglés optó por el establecimiento de un sistema capitalista de libre mercado. Liberalizar la economía tiene la implicación de que son los estratos más vulnerables los que soportan los costos de las fallas del mercado, es decir, los trabajadores,

\section{4}


los campesinos y las pequeñas y medianas empresas se vuelven vulnerables a los fracasos de esos mercados, lo que provoca o aumenta los niveles de desempleo, las desigualdades socioeconómicas y las enfermedades sociales como la pobreza y la violencia social. ${ }^{10}$

En este sentido, para Polanyi (2001), el problema principal con tal sistema económico es que el Estado ya no coordina la economía, lo que hace que pierda su fuerza para reaccionar en favor de la sociedad cuando el mercado falla. Esto provoca un clima de inestabilidad económica que pone en peligro el orden social pues cada grupo actúa por su cuenta para proteger sus intereses. La Gran Depresión, a finales de 1920, fue una de las consecuencias de dicho modelo de mercado. ${ }^{11} \mathrm{El}$ empobrecimiento de gran parte de la población inglesa y de la mayoría de los países más industrializados durante esa época llegó a tal punto que diversos grupos sociales se organizaron para exigir sus derechos, tales como bienestar económico y seguridad social (Marshall y Bottomore, 1992).

En consecuencia, el siglo XX estuvo marcado por estrategias de desarrollo dominadas por los Estados nación, los cuales se expandieron considerablemente, especialmente a través de la provisión de derechos sociales para personas pobres o vulnerables que culminaron en la creación del Estado de bienestar. ${ }^{12}$ En Latinoamérica, se formó lo que Barrientos (2004) denominó el Estado de bienestar conser-

10. Entre otras reformas del mercado de trabajo, el Gobierno británico redujo los subsidios de ayuda a los salarios (sólo se ayudaría a personas en situación de pobreza extrema),y presionó para la flexibilización en los procesos de contratación y separación del puesto con la idea de que las empresas nacionales redujeran sus costos de producción y pudieran competir internacionalmente. Polanyi (200I) ofrece una explicación detallada de las reformas y sus consecuencias.

I I. De hecho, Polanyi (200 I) señala que el clima de inestabilidad económica que se vivió en Europa, y en general en todo el mundo, provocó el estallido de la Primera Guerra Mundial.

12. Esping-Andersen (1990) explica de forma completa este proceso de formación del Estado de bienestar durante el siglo XX. 
vador-informal, que aunque se basaba en la protección del empleo como núcleo básico lo hacía únicamente respecto al sector formal (de los ámbitos industrial y gubernamental), por lo que dejaba desprotegidos a los trabajadores del sector informal. La formación del Estado de bienestar permitiría, hasta cierto punto, no solamente disminuir las desigualdades que había dejado la introducción de las máquinas de vapor en conjunto con la liberalización de la economía, sino también la adopción, a nivel mundial, de nuevas tecnologías.

Durante la etapa del Estado de bienestar, se presentaron en todo el mundo dos revoluciones tecnológicas más. De acuerdo a Simon (1987), algunas de las tecnologías a las que les fue adaptada la máquina o motor de vapor fueron la locomotora, el telar y, tal vez la más importante, el dínamo, que permitió generar electricidad. Una vez que se demostró que la electricidad era una fuente de energía menos costosa que las máquinas de vapor, se inició un periodo de cambio tecnológico tanto en las empresas públicas y privadas como en los hogares. Casi de manera simultánea sucedió otro cambio tecnológico: las dos guerras mundiales aceleraron el desarrollo de tecnologías para explotar el petróleo (motores de combustión interna, petroquímica, entre otras) y eso impactó también todos los sectores de la economía.

Sin embargo, a diferencia de lo que había sucedido durante la Revolución Industrial, la coordinación estatal de la economía en el Estado de bienestar permitió, entre otras cosas, crear diversos mecanismos de planeación y gestión de demanda de la nueva tecnología, tanto directamente a través del gasto en infraestructura, defensa y servicios públicos como indirectamente a través del empleo masivo del Gobierno (Perez, 1983). ${ }^{13}$

13. Perez (1983) y Barrientos (2004) mencionan, entre otros importantes cambios institucionales, la rápida expansión de la educación superior para alimentar el nuevo sistema tanto en el servicio público como en el privado, junto con la expansión de sistemas de salud pública y la aceptación de los sindicatos como representantes legales de los trabajadores, los cuales ayudaron a mejorar sus condiciones laborales y la planeación de las empresas.

\section{6}


En Latinoamérica, los préstamos internacionales y la exportación de materias primas como el petróleo y los minerales permitieron a los países en vías de desarrollo sostener el gasto del Estado de bienestar. Sin embargo, los países fueron incapaces de desarrollar algún sector, especialmente en el campo tecnológico, que les permitiera competir internacionalmente (Moreno-Brid, Partidas, y Ros, 2009). La región entró entonces en una de las peores crisis de la historia, lo que provocó grandes devaluaciones y altos niveles de inflación que a su vez desencadenaron un enorme aumento en los niveles de desempleo, desigualdad de ingresos y pobreza -entendida entonces como nutricional-. ${ }^{14}$

En este contexto, la llegada de los microchips, a inicios de los años setenta, significaría un nuevo cambio tecnológico: el inicio de la era de las TIC, en la que el internet, los teléfonos celulares, la computadora y las tabletas, entre otras tecnologías complementarias (softwares, aplicaciones, etc.), impactarían nuevamente la economía y las dinámicas al interior de los hogares. A la par, en México se iniciaría también un nuevo ajuste institucional que seguiría las recomendaciones del Fondo Monetario Internacional (FMI) y posteriormente del Banco Mundial (BM), como en la mayoría de los países latinoamericanos, con la intención de lograr estabilidad macroeconómica, aumentar el empleo y reducir la desigualdad de ingresos y la pobreza (Robertson, 2007).

Las reformas denominadas del consenso de Washington empezaron a ser implementadas a principios de los años ochenta en el país, y básicamente comprendían dos estrategias principales orientadas al mercado: la reducción del tamaño del Estado y la apertura de los sectores económicos

14. La tasa de inflación en la región aumentó de manera drástica de $17.3 \%$, en 1972, a I I 18.5\%, en 1990. A nivel país, los casos más dramáticos fueron los de Argentina, Bolivia, Brasil y Perú. En México, dicha tasa llegó a su cima en 1985, con $63.7 \%$. En cuanto a los índices de pobreza, la cantidad de personas en situación de pobreza extrema se duplicó a lo largo de los años ochenta, aumentando de 35.7 millones en 1980, a 73.I en 1990 (Spilimbergo, Luis Londoño, y Székely, 1997). 
con el fin de permitir que los mercados se ajustaran adecuadamente, en su propia dinámica, a las nuevas condiciones y desafíos que presentó el nuevo orden mundial.

En términos generales, se levantaron los controles de precios, se redujo el gasto social, se privatizaron las empresas estatales, se desreguló el sistema financiero y se redujeron las barreras arancelarias para los mercados nacionales. Paradójicamente, las condiciones socioeconómicas de los ciudadanos durante este periodo se agravaron debido en parte a los profundos recortes en el gasto social, lo que significó una regresión en el avance de los derechos sociales que se habían alcanzado en la primera mitad del siglo XX. ${ }^{15}$

A este respecto, Wacquant (2017) comenta que el problema con el Estado neoliberal es que opera siguiendo los preceptos del liberalismo económico, pero únicamente a favor de las élites económicas y políticas, mientras es fuertemente autoritario cuando se trata de lidiar con las consecuencias negativas de la apertura económica sobre los grupos más vulnerables (trabajadores, campesinos y pequeñas y medianas empresas). Esta contradicción no es más que la manipulación del marco institucional y organizativo de los Estados por parte de las élites, quienes se encargan de que este conjunto de instituciones se aplique de manera

I5. A pesar de que se estima que en la actualidad más del $50 \%$ de la población de América Latina trabaja en la informalidad o lo hace en microempresas con menos de cinco trabajadores, con algunas excepciones a nivel país como en Brasil, los Estados latinoamericanos gastan muy poco en políticas de empleo activas, destinadas a fomentar el desarrollo del capital humano, la creación de empleo o la intermediación entre los empleadores y los empleados (Schneider y Karcher, 20 I0; Bertranou y Maurizio, 20I I). Por otro lado, en Argentina, Brasil, Chile, Ecuador, Uruguay y Venezuela sólo se han desarrollado políticas de empleo pasivas, como el seguro de desempleo, aunque en la mayoría de dichos países la cobertura y los beneficios son muy bajos (Bertranou y Maurizio, 20I I). Más aún, la densidad sindical varió del 20 al 25\% en Brasil,Argentina y México, y del 10 al I5\% en Perú, Colombia y Chile, lo cual, aunado a la falta de políticas activas y pasivas de empleo, ha traído una alta inseguridad del ingreso pues la duración media en el empleo en el continente es de tres años, mientras que en los países desarrollados oscila entre cinco y 7.5 años (Schneider y Karcher, 2010).

\section{8}


imparcial e impersonal sólo en relación con los miembros de su coalición para perseguir sus objetivos económicos, políticos y sociales.

Por otro lado, la utilización de tecnologías como la robotización y la inteligencia artificial en las organizaciones públicas y privadas ha trastornado la estructura del mercado laboral y excluido incluso a personas con acceso a y apropiación de las Tic. De acuerdo al Foro Económico Mundial (World Economic Forum, 2016), entre 2015 y 2020 se perderán, a nivel mundial, más de 7.1 millones de empleos, principalmente en áreas administrativas y de oficina, y se crearán solamente alrededor de dos millones en los campos relacionados con la informática y las matemáticas y la arquitectura e ingeniería. La introducción de las TIC, en conjunto con la liberación de la economía, nuevamente ha dejado al descubierto la importancia de la intervención del Estado -a través de su marco institucional y organizacional- para prevenir y paliar los problemas sociales.

De acuerdo con Perez (1983), los cambios institucionales tal vez más importantes que se han presentado en este nuevo paradigma en los países en vías de desarrollo han sucedido en el mercado de trabajo, debido a la escasez de mano de obra calificada para el uso y apropiación de TIC. Entre ellos, se pueden mencionar la flexibilización de las horas de trabajo, la alfabetización digital, la creación de infraestructura para aumentar el acceso y uso de las TIC, entre otros. Sin embargo, como la misma Perez apunta, parecen ser cambios menores, ya que se requiere un ajuste institucional a mayor escala para emparejar el avance y desarrollo de la industria de las TIC con el régimen político actual. En este artículo, se propone que este ajuste institucional sea delineado por el sentido social de la apropiación de las TIC. 


\section{Apropiación de las tecnologías de la información y comunicación}

El termino apropiación social de las TIC ha sido ampliamente discutido (Rojas y Chalmers, 2009; Zejda, 2011; Zambrano, 2013; Pang, Zhang, Law, y Foo, 2016), sin embargo, es poca la claridad con la que se ha presentado, en especial respecto a su relación con la reducción de la pobreza. De acuerdo a Bar, Weber y Pisani (2016), la apropiación de la tecnología es un ciclo que inicia cuando las personas o usuarios deciden adoptar una y emplearla como soporte de sus actividades sociales o de negocios. Sin embargo, para que la tecnología evolucione de acuerdo a las necesidades de los usuarios y promueva el desarrollo social y económico, se debe ir más allá de su mera adopción.

La adopción es, entonces, la fase del ciclo en la cual los usuarios adquieren la tecnología y la utilizan con el propósito de ser más eficientes en sus actividades diarias. ${ }^{16}$ Como lo mencionan Bar, Weber y Pisani (2016), los usuarios realizan las mismas cosas utilizando la nueva tecnología, como hablar por teléfono celular en lugar del teléfono convencional, redactar textos en computadora y no en máquinas de escribir, entre otras. Es decir, lo que cambia es la

16. La adopción de la tecnología fue inicialmente analizada por Rogers (I962) en su teoría de la difusión de las innovaciones, que mencionaba que durante el proceso de difusión de la tecnología la percepción de los usuarios de cinco de sus características delimitaba su adopción. Dichas características son: I) ventaja sobre otras tecnologías, 2) compatibilidad con las necesidades de los usuarios, 3) complejidad de uso, 4) grado de experimentación que se puede realizar con la tecnología, y 5) grado en que las bondades de la tecnología son observables. Por su parte, Davis (1989) desarrolló su modelo de aceptación de la tecnología para entender las percepciones de los usuarios sobre por qué se adoptan las innovaciones tecnológicas. Como lo subrayaron Isaac, Besseyre Des Horts y Leclercq (2007), el problema de estas teorías iniciales sobre la adopción de la tecnología se encuentra en que no toman en cuenta el contexto estructural y cultural, los roles y las responsabilidades de los usuarios, y consideran la adopción como una decisión binaria: usarla o no. 
herramienta, no la práctica. ${ }^{17}$ No obstante, los individuos no siempre tienen la opción de aceptar o rechazar la tecnología: en muchas ocasiones, la elección que se hace no es una opción entre adoptar y no adoptar, sino entre hacerlo ahora o tomar la decisión más tarde si como usuarios pueden desarrollar diferentes tipos de uso, reinventarla o apropiarla (Hall y Khan, 2003; Isaac, Besseyre Des Horts, y Leclercq, 2007). ${ }^{18}$

La siguiente fase del ciclo ocurre cuando los usuarios empiezan a apropiarse de la tecnología, la experimentan, examinan sus posibilidades y modifican sus características para adaptarla a sus necesidades (Bar, Weber, y Pisani, 2016). En este sentido, la apropiación de la tecnología no sólo se refiere a la adquisición de conocimiento acerca de un objeto o de cómo hacer o aplicar algo con la tecnología, sino que la apropiación simultáneamente transforma al usuario (en sus conocimientos y habilidades) y a la tecnología (en sus propiedades) (Andrés, 2014). La apropiación de la tecnología podría definirse, entonces, como el proceso de adaptación de la tecnología a las actividades diarias de los

17. De acuerdo a Forman, Goldfarb y Greenstein (20I6), no sólo los individuos, sino también las organizaciones públicas, privadas, sociales o algún otro agente pueden adoptar tecnología, y esta puede referirse a un nuevo producto, servicio o innovación.

18. Orlikowski (1992) y Gerardine y Poole (1994) fueron de los primeros que presentaron definiciones sobre la adopción y apropiación de la tecnología, pero se limitaron a analizar dicho fenómeno en las organizaciones. Orlikowski (1992), por un lado, considera que la tecnología tiene un carácter dual y con flexibilidad interpretativa: dual porque es construida físicamente por actores en un contexto social específico y socialmente a través de los diferentes significados que cada actor social le confiere; flexibilidad interpretativa porque los actores son capaces de impactar el desarrollo de dicha tecnología. Gerardine y Poole (1994) desarrollaron la teoría de la estructuración adaptativa (AST, por sus siglas en inglés), que señala que la tecnología está envuelta en dos principales conceptos: su espíritu (la intención general de la tecnología) y su apropiación por parte de los individuos, esta última influenciada, por una parte, por las estructuras sociales de la tecnología (marco normativo) y sus características propias y, por otra, por las estructuras sociales en acción, es decir, los diferentes recursos del marco social (normas del grupo, ambiente, entre otros). 
usuarios con propósitos específicos: trabajo, comunicación, e incluso propósitos lúdicos.

Bar, Weber y Pisani (2016) explican que esta fase tiene como únicos límites la propia arquitectura de la tecnología impuesta por los proveedores, quienes al darse cuenta de las nuevas prácticas realizadas por los usuarios se posesionan nuevamente de la tecnología para reconfigurarla, de modo que el ciclo de adopción, apropiación y reposesión reinicia. El surgimiento de la banca móvil y el servicio de mensajes cortos en África son excelentes ejemplos de cómo se desarrolla este ciclo.

En el caso de la banca móvil, Bar, Weber y Pisani (2016) explican que los usuarios en dicho continente adoptaron inicialmente la telefonía de prepago para hablar por teléfono o enviar mensajes de texto, como en la mayor parte del mundo, pero con el tiempo recombinaron dos elementos del servicio: las recargas prepagadas y los mensajes de texto, al transferir o canjear crédito por dinero. Esto llevó a diversas compañías telefónicas a ofrecer servicios de banca móvil, tratando a los teléfonos como tarjetas de débito que permitían realizar compras, pagos o transferencias a otros teléfonos o a cuentas bancarias (Bar, Weber, y Pisani, 2016).

Por otro lado, el surgimiento del servicio de envío de mensajes cortos (SMS), en la década de 1980, permitió a los usuarios de teléfonos móviles desarrollar diferentes prácticas (servicios de alerta de emergencia, servicios de nómina, voto electrónico, entre otros), e incluso un leguaje especial, o texting (Acker, 2014; Bar, Weber, y Pisani, 2016). Los operadores, entonces, se reposesionaron del servicio y diversificaron su oferta al crear diferentes aplicaciones basadas en el mismo principio, como Twitter, Whatsapp, Wechat, Line, Viber, entre otras (Bar, Weber, y Pisani, 2016).

A este respecto, Bar, Weber y Pisani (2016) distinguen tres principales formas de apropiación de las TIC. La primera es el llenado de espacios tecnológicos que los proveedores 
dejan intencionalmente para que los usuarios personalicen la tecnología. Algunos ejemplos pueden ser el cambio en el protector de pantalla de la computadora o el celular, la instalación de nuevas aplicaciones, o hasta algunos que involucran cambios en la manera de socializar, como formar grupos en redes sociales para mantenerse en contacto o coordinar juntas o reuniones, entre otros. En general, la característica principal de las acciones que se realizan en esta primera forma de apropiación es que ninguna de ellas entra en conflicto con los proveedores de la tecnología.

La segunda forma es la recombinación de los componentes de la tecnología con la intención de generar nuevas prácticas que respondan mejor a las necesidades del usuario. ${ }^{19}$ Esta forma de apropiación representa una transformación más profunda. Algunos ejemplos podrían ser desarmar los elementos de los teléfonos celulares y recombinarlos, como cuando se intercambian sim cards o se modifica el teléfono para que soporte dos de esas tarjetas; fabricar antenas para mejorar la recepción o baterías alternas para recargar aparatos; utilizar el radio de los teléfonos en lugar de hacer llamadas para evitar un gasto en telefonía; la utilización de banca móvil, entre otros (Bar, Weber, y Pisani, 2016). A pesar de que esta forma de apropiación es un tanto más avanzada que la primera, estas prácticas tampoco entran en conflicto con los proveedores del servicio o del aparato tecnológico.

Finalmente, tomando el término que utilizara Schumpeter (1962) para describir los procesos de innovación en las economías liberales, Bar, Weber y Pisani (2016) señalan que la tercera forma de apropiación es una destrucción

19. Neüman (2008) señala que en el acto de apropiarse existe un ingrediente adaptativo, de conveniencia, y por otro lado, que es un acto de iniciativa propia, de propia autoridad. Es decir, que el acto de apropiarse no pasa por una concesión previa de lo apropiado ni es inspirado por terceros. Esta visión encaja con la segunda y tercera formas de apropiación que señalan Bar,Weber y Pisani (2016), ya que en la primera forma de apropiación esta sí es fomentada por el fabricante de la innovación tecnológica. 
creativa en la cual los usuarios, diseñadores, fabricantes y proveedores de servicio pelean por quién puede utilizar esa tecnología, a qué costo, en qué condiciones, con qué fin y con qué consecuencias. ${ }^{20}$ Algunos ejemplos podrían ser desbloquear el teléfono para evitar pagar impuestos, usar el bluetooth para compartir información sin conectarse a internet, la construcción de detonadores explosivos, entre otros. Los proveedores de la tecnología, entonces, tienen que decidir sobre suprimir una innovación que encuentran demasiado antagónica a sus objetivos, o adoptarla, aprender de ella e integrarla (Bar, Weber, y Pisani, 2016).

De acuerdo a Bar, Weber y Pisani (2016), el impacto socioeconómico más exitoso de las TIC se desarrollará donde exista un amplio margen para la experimentación y la apropiación impulsadas por el usuario, y donde las relaciones de cooperación entre los usuarios y los proveedores permitan el aprendizaje de la experimentación y la incorporación de los resultados dentro de nuevas innovaciones tecnológicas. Sin embargo, tendría que preguntarse para quién o en favor de quién se produciría dicho impacto socioeconómico.

Es en este punto donde el sentido social de la apropiación de las TIC se vuelve más importante, especialmente en países latinoamericanos, debido a las condiciones de desigualdad económica que estos presentan.

\section{Apropiación social}

La pregunta es ¿cómo lograr una apropiación de la tecnología con fines sociales? Más específicamente: ¿cómo se

20. Schumpeter (1962) señalaba que el sistema capitalista es por naturaleza un método o forma de cambio económico, ya que el impulso fundamental que lo mantiene en movimiento es la introducción de nuevos bienes de consumo, nuevos métodos de producción o transporte, nuevos mercados, nuevas formas de organización industrial, entre otros, que incesantemente revolucionan el marco institucional de la estructura económica desde dentro, incesantemente destruyendo el anterior e incesantemente creando uno nuevo.

\section{4}


logra la apropiación social de la tecnología para reducir la pobreza? Adam Smith (1976) argüía que en un sistema capitalista de libre mercado la desigualdad socioeconómica es inevitable, y que el Estado juega un papel relevante en la igualación de las condiciones socioeconómicas de la sociedad, puesto que una sociedad más productiva e igualitaria promovería el crecimiento económico. Smith (1976) se oponía a cualquier intervención o regulación que interfiriera con los mecanismos del mercado, siempre y cuando estos no dañaran a los pobres y exacerbaran las desigualdades socioeconómicas. El teórico ponía énfasis en la importancia de la intervención estatal para limitar el poder de los capitalistas, ya que estos podrían institucionalizar la pobreza y la desigualdad al obstaculizar el acceso a los servicios básicos como la educación.

Según Smith (1976), las diferencias en los niveles educativos traerían consigo la incapacidad de los ciudadanos para comprender tanto la configuración del mundo como la forma de mejorar sus condiciones. La distribución de la educación y capacitación tendría que ser el papel que el Estado juegue para ayudar a igualar las oportunidades de los pobres de tener un empleo y perseguir sus objetivos económicos, políticos y sociales.

Entonces, la provisión de educación para entender los cambios que produjeron las máquinas de vapor, y de capacitación para apropiarse de ellas, a través de un marco institucional, tendría que haber sido parte del camino que los países en vías de desarrollo siguieran cuando intentaron industrializarse durante la primera parte del siglo XX.

Con base en las ideas de Adam Smith, y a partir de los decepcionantes resultados de la liberalización económica de los países en desarrollo durante las décadas de los setenta y ochenta, Sen (1993) criticó la lógica de la liberalización de la economía en dichos países, que entendían el desarrollo en términos del crecimiento económico. 
Según Sen, medidas tales como el crecimiento del producto nacional bruto (PNB) o la industrialización no toman en cuenta los intereses de las personas por igualar sus oportunidades o contar con la libertad de perseguir el modo de vida que desean. En consecuencia, dicho autor sugirió la noción de capacidades como la medida que permite tener en cuenta dichos intereses y libertades, siendo esto tanto el objetivo primordial del desarrollo como el medio principal para lograr el crecimiento económico del país.

Esta concepción en torno a capacidades se centra en el individuo y está más preocupada por la eliminación de las desventajas o los elementos que impiden la libertad y creación de oportunidades (Anand y Sen, 1997). En este sentido, desde esta perspectiva la pobreza es una consecuencia del subdesarrollo humano, y el desarrollo humano se conceptualiza como la capacidad de las personas de hacer o ser las diversas funciones que puedan desempeñar (Sen, 1993). Por consiguiente, las funciones son diferentes de las mercancías: una mercancía es un objeto que una persona podría utilizar, como una casa o alimento, mientras que una función tiene que ver con que la persona viva de la manera que desea (Sen, 1993).

Por lo tanto, y en contraste con definiciones y mediciones anteriores de la pobreza que sólo hacían comparaciones entre niveles de ingreso o niveles de vida (consumo), desde el enfoque de capacidades el ingreso y el consumo son sólo instrumentos para alcanzar aquello que realmente importa: el rango de opciones y oportunidades para que cada persona pueda vivir de la manera que desea.

El logro de tal tipo de vida está determinado por un conjunto de capacidades que representan las oportunidades que tiene una persona para alcanzar la vida deseada: un aumento en las capacidades de cada persona mejorará su libertad de elegir su modo de vida. El desarrollo humano consiste, entonces, en la eliminación de las principales 
fuentes de no libertad en lo social, lo político y lo económico, como la pobreza, la falta de oportunidades económicas, la carencia de servicios básicos como la educación, la escasez de oportunidades para participar en el proceso de toma de decisiones públicas y la privación de la protección social. Eliminar estas fuentes de no libertad, entonces, reforzaría y potenciaría las capacidades de las personas (Sen, 1999).

En virtud de lo anterior, la pobreza es vista como la peor forma de privación humana, ya que puede implicar no sólo la ausencia de elementos necesarios para el bienestar material, sino también la negación de oportunidades para vivir de forma tolerable (Anand y Sen, 1997). El cambio en el paradigma del ingreso o el consumo a la noción de capacidades mejora la comprensión de la naturaleza y las causas de la pobreza, desplazando la atención de los medios (como el ingreso) a los fines que la gente persigue y las libertades para lograrlos (Sen, 1999). Dentro de este cambio en la noción, la pobreza representa la imposibilidad de llevar a cabo proyectos de vida autónomos, ya que los pobres carecen de igualdad de oportunidades para adquirir o acceder a los medios para realizar sus proyectos de vida.

Según Echeverría (2008), las TIC afectan una gama muy amplia de acciones humanas, no sólo a las comunicativas o a las que buscan y obtienen información y comunicación, es decir, transforman las capacidades al hacer posibles actividades antes inviables. En este sentido, como lo menciona Sen (2010), las TIC se han convertido en una cultura interactiva en el mundo, y la cuestión importante es cómo pueden hacer que las personas sean más funcionales, es decir, que puedan hacer -o ser- diversas actividades que les permitan alcanzar la vida que desean. Así, los pobres de la revolución tecnológica actual se caracterizan, entre otras cosas, por libertades limitadas en cuanto a la apropiación de las TIC con miras a alcanzar la vida que desean. 
El rol o papel del Estado, entonces, no debe limitarse a expandir las oportunidades en el acceso a las TIC, sino que debe potenciar las oportunidades de apropiación, es decir, de uso o utilización de estas tecnologías en las actividades diarias de las personas en situación de pobreza. Como lo señala Neüman (2008), para que se dé realmente la apropiación social es condición que el contacto con lo apropiable produzca un cambio, no sobre lo apropiable sino sobre las prácticas sociales asociadas con lo apropiable, y luego un paso más allá: los que se apropian deben poder regular el resultado del cambio en las prácticas sociales. Para ello, y de acuerdo a los niveles de apropiación de la tecnología anteriormente expuestos, se requiere la generación no sólo de habilidades digitales, sino de capacidades para las personas en situación de pobreza.

De acuerdo con Sen (2004), la definición de las capacidades necesarias para eliminar las fuentes de no libertad, como la pobreza, debería ser una cuestión empírica, resultante de la discusión y el razonamiento públicos sobre lo que debería incluirse como capacidad y por qué, todo dentro de un ambiente democrático que conduzca a una mejor comprensión del papel, alcance e importancia de las capacidades particulares. Esta aseveración ha influido distintos intentos de operacionalización, tanto empíricos como filosóficos. En términos generales, una persona es pobre si carece de capacidades básicas en tres dimensiones: esperanza de vida al nacer, años de escolaridad e ingreso nacional bruto per cápita. Como resultado de las privaciones a estas capacidades básicas, los pobres ven limitadas sus oportunidades de alcanzar metas sociales, políticas y económicas.

Todos estos desarrollos han llevado a un renovado énfasis en la importancia del papel del Estado como actor crucial para el alivio de la pobreza a través de la cobertura de servicios básicos como la educación. La búsqueda estaría en 
que las personas pobres puedan obtener ingresos sostenidos en el mercado laboral para salir de su situación.

Sin embargo, el enfoque de capacidades no es suficiente para explicar las causas o consecuencias de la distribución o redistribución desigual de los servicios y recursos públicos que afecta a los pobres. Esto hace que el paradigma de capacidades sea difícil de emplear en contextos de alta desigualdad socioeconómica, como en América Latina y algunos países de África donde, entre otros factores importantes, la debilidad institucional de los Estados es una de las causas clave que impiden la igual distribución de recursos y servicios que podrían tener un impacto en el alivio de la pobreza.

De hecho, Gillwald (2010) argumenta que la experiencia de los países escandinavos, los tigres asiáticos y Estados Unidos de América (EUA) indica que el potencial social de las TIC está condicionado por los sistemas institucionales de financiación, regulación y de incentivos y sanciones del Estado. North, Wallis y Weingast (2007) explican que sin instituciones imparciales e impersonales que permitan a toda la población el acceso, adopción y apropiación de las TIC, las élites pueden adoptar la tecnología de una manera selectiva sin necesidad de permitir el despliegue de la densidad organizacional requerida para el desarrollo de la tecnología propia por así convenir a sus intereses.

Por ejemplo, la introducción de la radio y luego de la televisión en la primera mitad del siglo XX facilitó mucho a las personas en los países en vías de desarrollo obtener información, lo que a su vez les permitió comparar sus circunstancias y el desempeño de sus líderes. Sin embargo, estos mismos medios han facilitado que las élites difundan sus opiniones ante las masas y controlen su percepción del rendimiento de las organizaciones del Estado (North, Wallis, y Weingast, 2007). En el caso de las TIC, las redes sociales fueron el principal medio de comunicación -interactiva-que se utilizó durante los sismos ocurridos en México 
en septiembre de 2017, lo que aumentó potencialmente la coordinación entre los ciudadanos. No obstante, algunos Gobiernos han intentado restringir el acceso a internet y hacer un seguimiento de quiénes y con qué fines lo utilizan, lo que obstaculizaría la organización de la sociedad civil.

\section{Conclusiones}

El marco de análisis presentado sugiere que el surgimiento de innovaciones tecnológicas de la magnitud de las TIC hace imprescindible su difusión para que puedan ser adoptadas por organizaciones públicas, privadas, sociales, individuos o cualquier otro agente y, posteriormente, se lleve a cabo su apropiación. A lo largo de este ciclo, se requiere de un proceso de creación destructiva del marco institucional -internacional y nacional- que soporta tanto la estructura económica del Estado como el desarrollo, adopción y apropiación de las innovaciones tecnológicas. La interdependencia que se crea entre estas variables convierte a las innovaciones tecnológicas en parte del marco institucional del Estado cuando se adoptan a gran escala.

En este sentido, la reducción de problemas sociales como la pobreza a través de las TIC es una función del Estado, porque el propio Estado puede crear o reproducir estas condiciones por medio de su conjunto institucional y organizativo. Por ello, se requieren análisis institucionales de los Estados que permitan coadyuvar en la construcción de capacidades -organizacionales y humanas-para promover la apropiación social de las TIC para reducir la pobreza. Esto especialmente en Latinoamérica, cuyos Estados han imitado los procesos de ajuste institucional de otras sociedades desde la primera Revolución Industrial. 
Acker, A. (20I4). "The Short Message Service: Standards, Bibliografía Infrastructure and Innovation". Telematics and Informatics, 3 I (4), 559-568. Recuperado de: https://www.sciencedirect.com/science/article/pii/S07365853 I 4000 I5X

Anand, S., y Sen, A. (1997). "Concepts of Human Development and Poverty:A Multidimensional Perspective”, en R.Jolly (coord.), Poverty and Human Development: Human Development Papers 1997 (pp. I-20). Nueva York: United Nations Development Program.

Andrés, G. D. (20|4). “Una aproximación conceptual a la 'apropiación social' de TIC”. Questión, I (43), I5. Recuperado de:http://perio.unlp.edu.ar/ojs/index.php/question/ article/view/2227

Bar, F., Weber, M. S., y Pisani, F. (20I6). “Mobile technology appropriation in a distant mirror: Baroquization, creolization, and cannibalism”. New Media \& Society, I8(4), 617-636. doi: 10.1 177//46|4448|6629474

Barrientos, A. (2004). “'Latin America: a liberal-informal welfare regime?", en I. Gough, G.Wood,A. Barrientos, P. Bevan, P. Davis, y G. Room (eds.), Insecurity and welfare regimes in Asia, Africa and Latin America: Social policy in development contexts (pp. 121-168). Cambridge: Cambridge University Press.

Bertranou, F. M., y Maurizio, R. (20I I). The Role of Labour Market and Social Protection in Reducing Inequality and Eradicating Poverty in Latin America. Recuperado de: http://mpra.ub.uni-muenchen.de/39843/I/MPRA_ paper_39843.pdf

Boncheva, A. I., Licona Michel, Á., Loaiza Becerra, M., Mendoza Martínez, E., Rangel Delgado,J. E., y Uscanga Prieto, C. (20I6). "Las políticas gubernamentales de ciencia y tecnología en el Asia Pacífico en la posguerra: los casos de Japón y Corea del Sur". PORTES, revista mexicana de estudios sobre la Cuenca del Pacífico, I0(20), I05-136. 
Bibliografía
Recuperado de: http://revistasacademicas.ucol.mx/ index.php/portes/article/view/I 282

Bresnahan,T. F. (2002). “Prospects for an Information-Technology-Led Productivity Surge”. Innovation Policy and the Economy, 2, I 35-161. Recuperado de: http://www.nber. org/chapters/c10787.pdf

Bresnahan,T. F. (20I0).“General Purpose Technologies”, en B. H. Hall,y N. Rosenberg (eds.), Handbook of the Economics of Innovation. Vol. 2 (pp.76I-79I). North-Holland: Elsevier. Bresnahan, T. F., y Trajtenberg, M. (1995). “General purpose technologies. 'Engines of growth'?”. Journal of Econometrics, 65(I), 83-I08. Recuperado de: https://www.sciencedirect.com/science/article/pii/03044076940 I 598T

David, P. ( 1989). Computer and dynamo: The modern productivity paradox in a not-too distant mirror. Recuperado de: https:// EconPapers.repec.org/RePEc:wrk:warwec:339

Davis, F. D. (1989). "Perceived Usefulness, Perceived Ease of Use, and User Acceptance of Information Technology”. MIS Quarterly, 13(3), 319-340. Recuperado de: https://www.researchgate.net/profile/Fred_Davis2/ publication/200085965_Perceived_Usefulness_Perceived_Ease_of_Use_and_User_Acceptance_of_Information_Technology/links/54ad66dc0cf24aca I c6f3765.pdf

Echeverría, J. (2008). “Apropiación social de las tecnologías de la información y la comunicación". Revista iberoamericana de ciencia, tecnología y sociedad, 4, I7|- I82. Recuperado de:http://www.scielo.org.ar/pdf/cts/v4n I0/ v4nI0all.pdf

Esping-Andersen, G. (1990). The three worlds of welfare capitalism. Cambridge: Polity Press.

Evans, P., y Sewell,W. H. (2013). "Neoliberalism: Policy Regimes, International Regimes, and Social effects", en P.Hall, y M. Lamont (eds.), Social Resilience in the Neo-Liberal Era (pp. 35-68). Nueva York: Cambridge University Press. 
Fagerberg, J., Srholec, M., y Verspagen, B. (20 I0).“Innovation Bibliografía and Economic Development", en B. H. Hall,y N. Rosenberg (eds.), Handbook of the Economics of Innovation. Vol. 2 (pp. 833-872). North-Holland: Elsevier.

Forman, C., Goldfarb, A.,y Greenstein, S. (2016).“Technology Adoption”, en M.Augier, y D.J.Teece (eds.), The Palgrave Encyclopedia of Strategic Management (pp. I-5). Londres: Palgrave Macmillan.

Freeman, C., y Perez, C. (1988). "Structural Crises of Adjustment: Business Cycles and Investment Behaviour", en G. Dosi, C. Freeman, R. R. Nelson, G. Silverberg, y L. L. G. Soete (eds.), Technical change and economic theory (pp. 87I-900). Londres: Pinter.

Galperin, H. (2010). “Goodbye Digital Divide, Hello Digital Confusion? A Critical Embrace of the Emerging ICT4D Consensus". Information Technologies \& International Development, 6(SE), 53-55. Recuperado de: http://itidjournal. org/itid/article/viewFile/623/263

Galperin, H., y Fernanda Viecens, M. (20I7). “Connected for Development? Theory and evidence about the impact of Internet technologies on poverty alleviation". Development Policy Review, 35(3), 3 I 5-336. doi: I 0. I I I I/ dpr. 12210

Gerardine, D.,y Poole, M.S. (1994)."Capturing the Complexity in Advanced Technology Use: Adaptive Structuration Theory". Organization Science, 5(2), I 2 I - I 47. Recuperado de: http://eli.johogo.com/Class/Gerry.pdf

Gerschenkron,A. (1962). Economic backwardness in historical perspective: A book of essays. Cambridge: Belknap Press of Harvard University Press.

Gillwald,A. (20I0)."The Poverty of ICT Policy, Research, and Practice in Africa". Information Technologies \& International Development, 6(SE), 79-88. Recuperado de: http://dev. itidjournal.org/index.php/itid/article/viewFile/628/268 
Bibliografía
Hall, B., y Khan, B. (2003). Adoption of New Technology. Recuperado de: https://EconPapers.repec.org/RePEc:cdl: econwp:qt3wg4p528

Isaac, H., Besseyre Des Horts, C. H., y Leclercq, A. (2007). Adoption and appropriation: Towards a new theoretical framework. An exploratory research on mobile technologies in French companies. Recuperado de: https://halshs. archives-ouvertes.fr/halshs-00I55506

Jovanovic, B.,y Rousseau, P.L. (2005).“'General Purpose Technologies”, en P.Aghion, y S. N. Durlauf (eds.), Handbook of Economic Growth. Vol. I (pp. I I8I-1224). Ámsterdan: Elsevier.

Lopez-Aymes,J.F.(2010).“Automobile, Information and Communication Technology and Space Industries as Icons of South Korean Economic Nationalism". Pacific Focus, 25(2), 289-3 I2. doi: I0. I I I I/j. I976-5 I I8.20I0.01045.x

Manca, F. (20I0). "Technology catch-up and the role of institutions”. Journal of Macroeconomics, 32(4), I 04 I- I 053. Recuperado de:https://www.sciencedirect.com/science/ article/pii/SO I640704I 0000637

Marshall,T. H., y Bottomore,T. (1992). Citizenship and Social Class. Londres: Pluto Press.

Moreno-Brid, J. C., Pardinas, J. E., y Ros, J. (2009).“Economic development and social policies in Mexico". Economy and Society, 38(I), 154-I 76. Recuperado de: http://media. library.ku.edu.tr/reserve/resspring I 7/Int1532_ZOnis/9_ Required.pdf

Neüman, M. I. (2008). "Construcción de la categoría ‘apropiación social’”. Quórum académico, 5(2), 67-98. Recuperado de: http://www.redalyc.org/articulo. oa? id $=1990 \mid 6835004$

Nord,J. H., Riggio, M.T., y Paliszkiewicz,J. (2017)."Social and Economic Development through Information and Communications Technologies: Italy". Journal of Computer Information Systems, 57(3), 278-285. Recuperado de: https:// 
search.proquest.com/openview/42374cb62040ec623 Bibliografía $50855 \mathrm{cf0b} 73638 \mathrm{~b} / \mathrm{I}$ ?pq-origsite $=$ gscholar $\& \mathrm{cbl}=48936$

North, D. C. (1994). Institutions and Productivity in History. Recuperado de: http://desarrl.handle.net/ I0535/438 I North, D. C., Wallis, J. J., y Weingast, B. R. (2007). Limited Access Orders In The Developing World: A New Approach To The Problems Of Development. Policy Research Working Paper no. WPS 4359. Washington:World Bank.

Orlikowski,W.J. (1992)."The Duality of Technology: Rethinking the Concept of Technology in Organizations”. Organization Science, 3(3), 398-427. Recuperado de: https:// dourish.com/ dourishc/classes/readings/OrlikowskiDualityOfTechnology-OrgSci.pdf

Pang, N., Zhang, X., Law, P.W., y Foo, S. (2016). “Coping with Ageing Issues:Adoption and Appropriation of Technology by Older Adults in Singapore", en J. Zhou, y G. Salvendy (eds.), Human Aspects of IT for the Aged Population. Healthy and Active Aging: Second International Conference, ITAP 20 16, Held as Part of HCl International 2016 Toronto, on, Canada, July I 7-22, 201 l 6. Proceedings. Part II (pp. 364374). Toronto: Springer International Publishing.

Perez, C. (1983). "Structural change and assimilation of new technologies in the economic and social systems". Futures, I5(5), 357-375. Recuperado de: https://www.sciencedirect.com/science/article/pii/00I 6328783900502

Perez, C. (20I3). "Financial bubbles, crises and the role of government in unleashing golden ages", en A. Pyka, y H. P. Burghof (eds.), Innovation and Finance (pp. I I-25). Londres: Routledge.

Polanyi, K. (200I). The great transformation. Boston: Beacon Press.

Qiu, R., y Cantwell, J. (20I6).“The international geography of general purpose technologies (GPTs) and internationalisation of corporate technological innovation". Industry 
Bibliografía and Innovation, 25(I), I-24. doi: I0.I080/I36627I6.20 16.1264065

Ristuccia, C., y Solomou, S. (20 I 0). General Purpose Technologies and Economic Growth:Electricity Diffusion in the Manufacturing Sector Before WWII. doi: I0.I 7863/CAM.5549

Robertson, R. (2007). "Trade and Wages:Two Puzzles from Mexico”. The World Economy, 30(9), I378-1398. doi: I0.1 I I I/j. I 467-9701.2007.01048.x

Rogers, E. M. (1962). Diffusion of innovations. Nueva York: Free Pr. of Glencoe.

Rojas, J.,y Chalmers, M. (2009).“The Appropriation of Information and Communication Technology:A Cross-Cultural Perspective", en J.A. Jacko (ed.), Human-Computer Interaction. New Trends: I 3th International Conference, $\mathrm{HCl}$ International 2009, San Diego, CA, USA, July 19-24, 2009. Proceedings. Part I (pp. 687-696). Berlín: Springer.

Rosenberg, N.,y Trajtenberg, M. (2004).“A General-Purpose Technology at Work: The Corliss Steam Engine in the Late-Nineteenth-Century United States". The Journal of Economic History, 64(I), 6I-99. Recuperado de: https:// www.tau.ac.il/ manuel/pdfs/A_GPT_At_work_The Corliss_SE.pdf

Rousseau, P. L. (2008). “General Purpose Technologies”, en S. N. Durlauf, y L. E. Blume (eds.), The New Palgrave Dictionary of Economics (pp. I-6). Basingstoke: Palgrave Macmillan.

Saviotti, P. P. (2005). "On the Co-Evolution of Technologies and Institutions”, en M.Weber,y J. Hemmelskamp (eds.), Towards Environmental Innovation Systems (pp. 9-3I). Berlín: Springer.

Schneider, B. R., y Karcher, S. (2010). "Complementarities and continuities in the political economy of labour markets in Latin America". Socio-Economic Review, 8(4), 623-65I. Recuperado de:https://academic.oup.com/ser/ article/8/4/623//676835 
Schumpeter, J.A. (1962). Capitalism, socialism, and democracy. Bibliografía Nueva York: Harper y Amp.

Sen, A. (1993). “Capability andWell-Being”, en M. Nussbaum, y A. Sen (eds.), The Quality of Life (pp. 30-53). Oxford: University Press.

Sen, A. (1999). Development as freedom. Oxford: Oxford University Press.

Sen, A. (2004). "Capabilities, Lists, and Public Reason: Continuing the Conversation". Feminist Economics, 10(3), 77-80. doi: I0.1080/I3545700420003 I 5 | 63

Sen, A. (2010). "Reflections From and On The Forum The Mobile and the World". Information Technologies \& International Development, 6(SE), I-3. Recuperado de: http:// itidjournal.org/index.php/itid/article/view/6 /4/254

Simon, H.A. (1987). "The steam engine and the computer: What makes technology revolutionary". EDUCOM Bulletin, 22, 2-5. Recuperado de: http://digitalcollections.library. $\mathrm{cmu}$.edu/awweb/awarchive? type $=$ file\&item $=34057$

Smith, A. (1976). An inquiry into the nature and causes of the wealth of nations. Oxford: Clarendon Press.

Spilimbergo,A., Luis Londoño, J., y Székely, M. (1997). Income Distribution, Factor Endowments, and Trade Openness. Working Paper Series. Núm. 356.Washington: Oficina del Economista Principal, Banco Interamericano de Desarrollo.

Standing, G. (2010). Work after globalisation: building occupational citizenship. Cheltenham, Reino Unido-Northampton, Estados Unidos: Edward Elgar.

Unión Internacional de Telecomunicaciones (2017). Measuring the Information Society Report 2017. Recuperado de:https://www.itu.int/en/ITU-D/Statistics/Documents/ publications/misr20I7/MISR20I7_Volumel.pdf

Wacquant, L. (2017). "The punitive regulation of poverty in the neoliberal age”, en V. Dashavantha Reddy, K.Venkateswara Rao, y K. Rama Krishna (eds.), Insights on Global 
Bibliografía
Challenge and Opportunities for the Century Ahead (pp. 85-88). India: BS Publications.

World Economic Forum (2016). The Future of Jobs. Employment, Skills and Workforce Strategy for the Fourth Industrial Revolution. Recuperado de: http://www3.weforum.org/ docs/WEF_Future_of_Jobs.pdf

Zambrano, N. (20I3). Beyond digital inclusion: Social appropriation of information and communication technologies. An experience in Venezuela: Infocentros. doi: 10.1109/ CLEI.2013.66706I5

Zejda, D. (20I I).“The Model of Appropriation: Contribution of Rational Choice Theory and Cognitive Science to a Better Technology". Ponencia presentada en el Proceedings of the 201 I Seventh International Conference on Intelligent Environments. Nottingham, Reino Unido. doi: I0.1 I09/|IE.20 I I.58 